\title{
Microfabrication of near-field optical probes
}

\author{
A. G. T. Ruiter, M. H. P. Moers, and N. F. van Hulst \\ Department of Applied Physics, MESA Research Institute, University of Twente, Enschede, The Netherlands \\ M. de Boer \\ Department of Electrical Engineering, MESA Research Institute, University of Twente, Enschede, The \\ Netherlands
}

(Received 19 May 1995; accepted 19 January 1996)

\begin{abstract}
Near-field optical microscopy generally uses a tapered optical fiber, which is metal coated, to form a sub-wavelength sized light source. Here, a technique for the fabrication of a new type of probe is described. The new design is based on atomic force microscope probes and consists of a silicon nitride cantilever with a solid transparent conical tip. The probes are made using micromechanical techniques, which allow batch fabrication of the probes. A near-field scanning optical microscope system was built to test the probes. This system features force detection by a beam deflection technique and subsequent force feedback together with a conventional optical microscope. A major advantage of the apparatus is the ease at which images are obtained. Results on a test sample show that an optical resolution of $300 \mathrm{~nm}$ can be obtained together with a simultaneous height image.

(C) 1996 American Vacuum Society.
\end{abstract}

\section{INTRODUCTION}

Near-field scanning optical microscopy (NSOM) is a technique in which an ultrahigh-resolution optical image of a sample can be obtained. ${ }^{1-7}$ In traditional optical microscopy, resolution is restricted by the diffraction limit resulting in a maximum achievable resolution of about half the optical wavelength. By using an aperture significantly smaller than the diffraction limit a small light source, or detector, can be created. ${ }^{8,9}$ When scanning this sub-wavelength light source or detector over a sample surface, an optical image can be obtained, with a resolution well below the diffraction limit.

Over the last few years, developments in the NSOM technique have been moving rapidly, which has already resulted in the development of the first commercial system. ${ }^{10}$ At present, most of the systems in use apply a sub-wavelength light source to illuminate the sample. The advantage is that only a small part of the sample is illuminated and optical damage to the sample, e.g., heating or bleaching, is minimal. The aperture light source consists of an optical fiber with one tapered end which has a tip size of about $50 \mathrm{~nm}$. Aluminum is evaporated onto the fiber from an angle perpendicular to the fiber axis while rotating the fiber, resulting in coated sidewalls of the fiber while the tip end remains uncoated. The metal layer is to discriminate far-field contributions in order to create a sub-wavelength aperture at the end of the fiber.

The fiber is scanned over the sample while simultaneously detecting the light transmitted through the sample. In order to obtain a high-resolution image, the fiber must be scanned in close proximity to the sample, usually at a distance much smaller than $50 \mathrm{~nm}$. This requirement has led to the development of the shear force distance regulation technique in which the fiber is laterally vibrated at its resonance frequency. ${ }^{11,12}$ Due to forces between the fiber and the sample the amplitude changes while approaching the sample. In literature several approach curves have been reported with feedback set points ranging from 1 to $20 \mathrm{~nm} \cdot{ }^{7,11-17}$ The origin and amplitude of the forces causing the amplitude of the shear force vibration to decrease are not known. They could be caused by a chemical effect between the probe and the sample or be related to frictional forces. In that case, tipsample distance varies on different areas of the substrate and therefore influences the NSOM operation. Operation of the shear force mechanism in liquid has yet to be demonstrated in imaging.

One of the drawbacks using these systems is that the operator has to be an experienced user and familiar with the technology in order to use the microscope. Especially, one has to be careful to avoid the tip crashing into the sample. Because of the high stiffness of the fiber in the direction perpendicular to the sample and the small operating distance from the sample, delicate approaching and vibration free operation conditions are essential. To overcome these problems, we have developed a new type of NSOM probe.

The design of this probe is based on the standard cantilever-type probes used in atomic force microscopy $(\mathrm{AFM})^{18}$ which has the advantage that tip damage is minimal due to the cantilever's small spring constant in the direction perpendicular to the sample. Applying the standard AFM probes in NSOM ensures an easy operable microscope which combines optics with force detection and thereby enabling all AFM contrast mechanisms, e.g., contact, noncontact, lateral force, tapping, and adhesion mode, to be combined with NSOM. The standard AFM probes have also been used in photon scanning tunneling microscopy (PSTM) ${ }^{19-24}$ and also earlier investigations ${ }^{25}$ have shown the possibilities of these probes. However, the commercially available AFM probes with pyramidal tips are not ideally suited for NSOM usage. The tips on these probes are made of the same material as the cantilever, where pyramidal pits in a crystalline silicon wafer are used as a mold to shape the tips, resulting in a hollow $\mathrm{Si}_{3} \mathrm{~N}_{4}$ tip. This is one of the reasons why polarization con- 


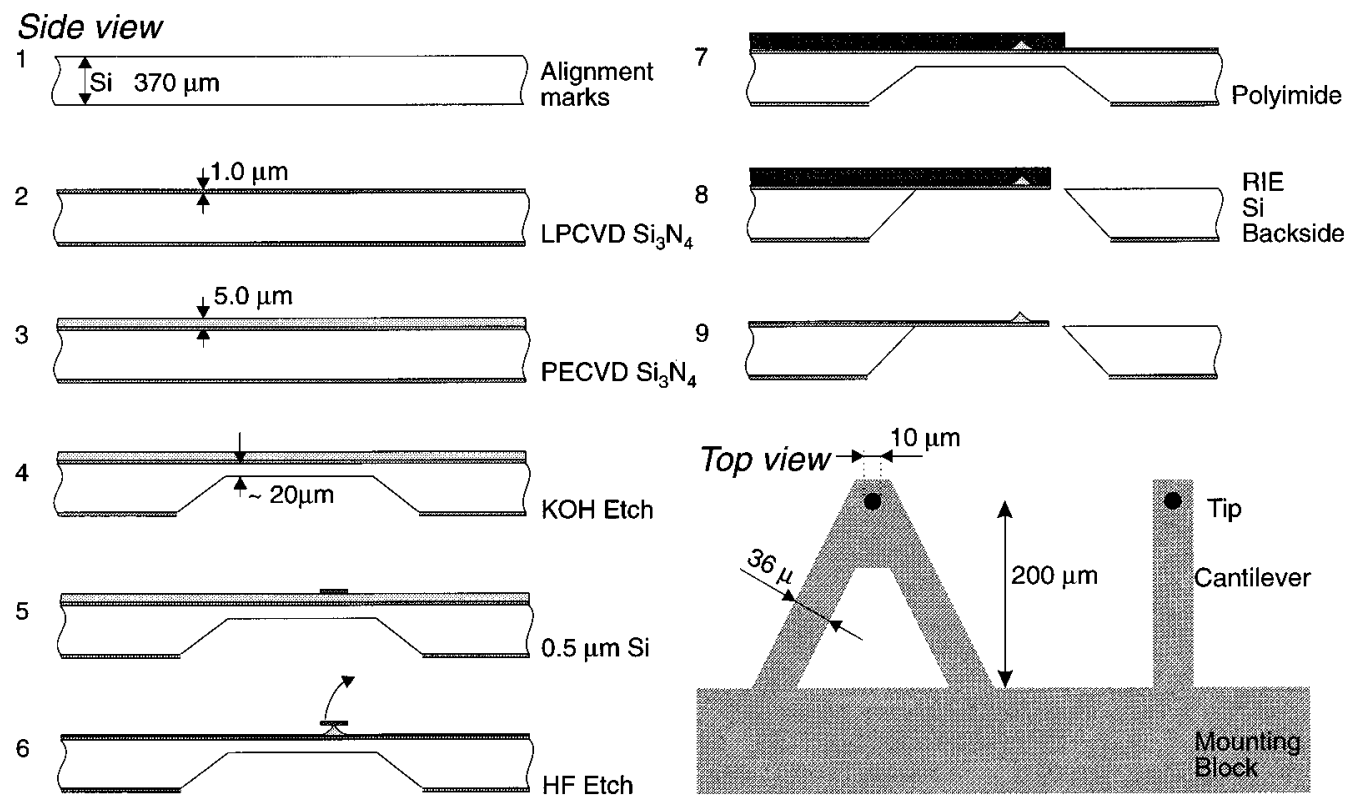

FIg. 1. Fabrication process; (1) 3 in. Si-wafer with alignment marks, (2) $1.0 \mu \mathrm{m} \mathrm{LPCVD} \mathrm{Si} \mathrm{N}_{4}$ deposition for cantilever, (3) $5.0 \mu \mathrm{m} \mathrm{Si} \mathrm{N}_{4} \mathrm{PECVD} \mathrm{deposition}$ for the tip, (4) $\mathrm{KOH}$ etch of $\mathrm{Si}$, (5) $0.5 \mu \mathrm{m}$ poly-silicon sputtered and patterned in small $10 \mu \mathrm{m}$ size disks, (6) isotropic $\mathrm{HF}$ etching of $\mathrm{Si}_{3} \mathrm{~N}_{4}$ to form the tip, (7) spinning of polyimide covering the tip, (8) dry etching of $\mathrm{Si}_{3} \mathrm{~N}_{4}$ and dry etching of silicon backside, (9) removal of the polyimide.

trast has not yet been shown in PSTM. The mechanism with which light, focused from the base side of the tip, is coupled into the hollow tip is less efficient than when a solid tip would be used. These considerations have led to the development of a cantilever probe with a solid transparent tip.

\section{FABRICATION OF NSOM PROBES}

The newly developed probes are made of silicon nitride using a $3 \mathrm{in}$. silicon wafer for mounting. The major layout of the wafer is such that small 2 by $3 \mathrm{~mm}$ silicon mounting blocks are formed, each having four cantilevers attached to it. Figure 1 shows the fabrication process in short. The cantilever is formed by a $1.0 \mu \mathrm{m}$ silicon rich $\mathrm{Si}_{3} \mathrm{~N}_{4}$ layer deposited by low-pressure chemical vapor deposition (LPCVD) at $850{ }^{\circ} \mathrm{C}, 200$ mTorr using a $\mathrm{SiCl}_{2} \mathrm{H}_{2} / \mathrm{NH}_{3}$ mixture $(80 \% / 20 \%)$ on a 3 in. Si wafer with alignment marks (see Fig. 1, steps 1 and 2). The tips are formed out of a $5.0 \mu \mathrm{m} \mathrm{Si}_{3} \mathrm{~N}_{4}$ layer deposited by plasma enhanced chemical vapor deposition (PECVD) [see Fig. 1, step 3]. The difference in density between the LPCVD and PECVD $\mathrm{Si}_{3} \mathrm{~N}_{4}$ layers results in an etch speed difference between the two layers in HF etchant. The PECVD layer etches over 20 times faster than the LPCVD layer effectively making the LPCVD layer an etch stop layer. The backside of the silicon wafer is etched anisotropically using potassium hydroxide $(\mathrm{KOH}, 50 \%)$ where a $20 \mu \mathrm{m}$ thick layer is preserved giving the wafer some rigidity for handling during the rest of the process, (see Fig. 1, step 4). In the $\mathrm{KOH}$ etching process breaking trenches are etched in the silicon for removal of the individual tipmounting blocks.

Polycrystalline silicon $(0.5 \mu \mathrm{m})$ is sputtered onto the PECVD layer which is used as a mask for the tip etching step. The poly-silicon is patterned into small disks with a diameter of $10 \mu \mathrm{m}$ (see Fig. 1, step 5). When etched in HF $\left(50 \%\right.$ at $\left.25^{\circ} \mathrm{C}\right)$, the $\mathrm{Si}_{3} \mathrm{~N}_{4}$ etches isotropically and the etching is stopped when the silicon disk is etched free, (see Fig. 1 , step 6). In order to control the process, disks have been patterned on the $\mathrm{Si}_{3} \mathrm{~N}_{4}$ layer with diameters ranging in size from 4 to $20 \mu \mathrm{m}$, so they will be etched free in order of size. A thick layer of polyimide is used to mask the tip and the cantilever (see Fig. 1, step 7). The cantilever shape is etched using reactive ion etching (RIE) in a $\mathrm{CHF}_{3} / \mathrm{O}_{2}$ plasma $(83 \%$ / $17 \%$ ). The silicon backside is etched open using RIE in a $\mathrm{SF}_{6} / \mathrm{O}_{2}$ plasma (75\%/25\%) (see Fig. 1, step 8). After stripping the polyimide, (see Fig. 1, step 9), the process is completed and the individual mounting blocks with tips can be broken from the wafer.

Figure 2 contains the results of the process showing the isotropic HF-etched tips from several disk sizes. It can be seen that the $10 \mu \mathrm{m}$ disk provides a conical tip while the larger disks are still attached to the PECVD layer. The smaller disks were all detached from the surface. Figure 2(d) shows the V-shaped cantilever with conical tip. The wafer, photographed by a scanning electron microscope (SEM), contained 448 cantilevers on 112 mounting blocks, clearly showing the benefit of batch fabrication of the probes.

The aperture in the tips is created by evaporating $\mathrm{Al}$ on the sidewalls of the tip, done by evaporating $\mathrm{Al}$ at an angle of about $85^{\circ}$ normal to the cantilever while rotating the tips. By this technique the layer thickness on the sidewalls of the tips is considerably larger than on the tip end. It also ensures that the layer thickness on the cantilever will be minimal, enabling normal optical imaging through the cantilever for alignment of sample and tip and avoiding bending of the 


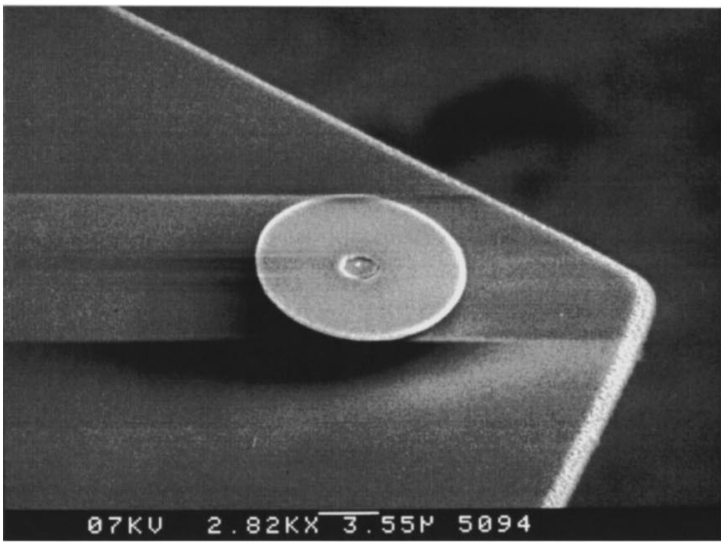

(a)

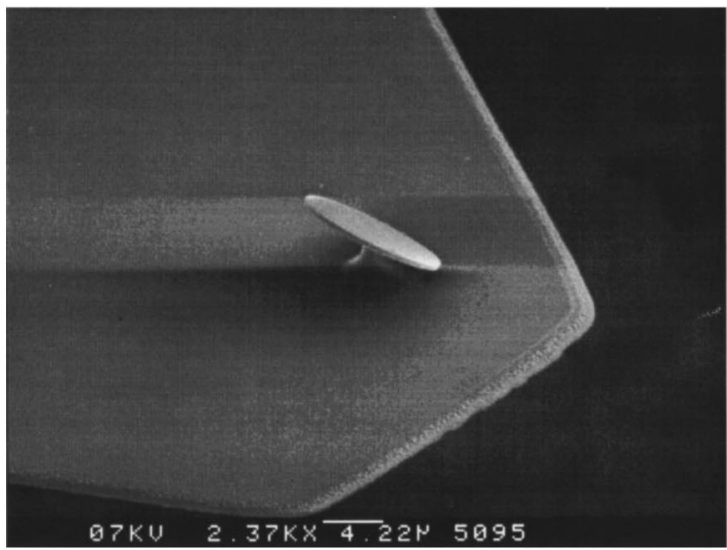

(b)

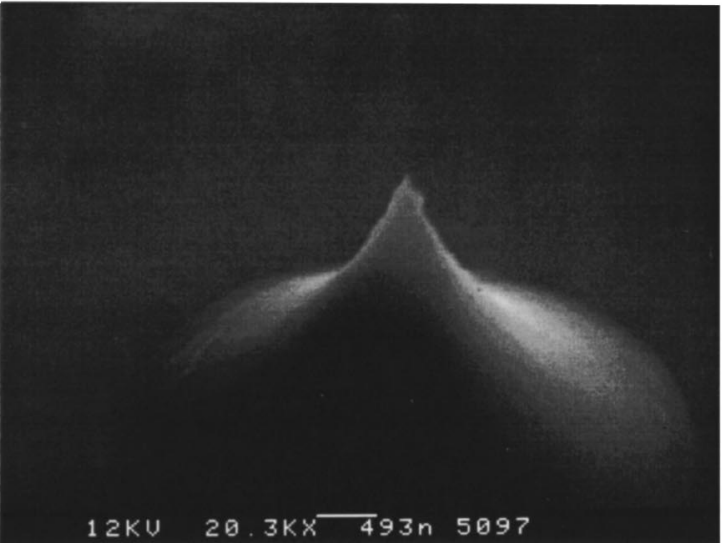

(c)

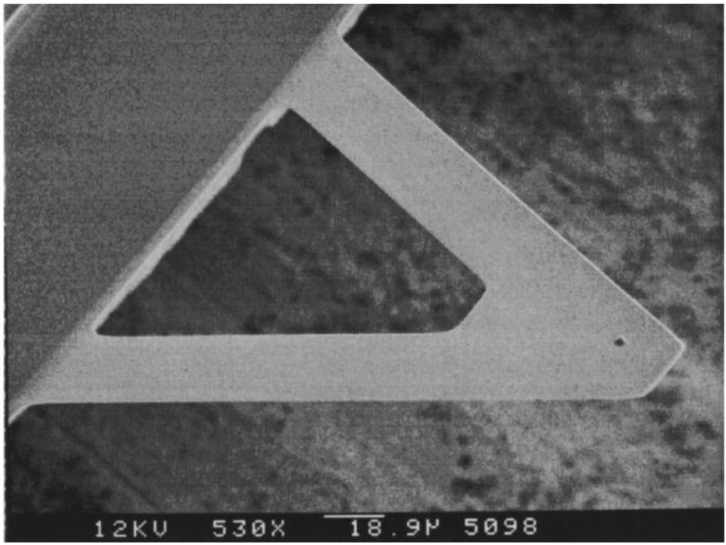

(d)

FIG. 2. (a) Result at a $16 \mu \mathrm{m}$ disk when the $10 \mu \mathrm{m}$ disk is etched free; (b) a $12 \mu \mathrm{m}$ disk; (c) tip from a $10 \mu \mathrm{m}$ disk; (d) the (c) image zoomed out showing the V-shaped cantilever with conical tip.

cantilever due to stress in the metal layer. On the tip used in this article, $\mathrm{Al}$ was evaporated at an angle of $83^{\circ}$, resulting in a $150 \mathrm{~nm}$ thick layer surrounding the tip and a $50 \mathrm{~nm}$ layer on the cantilever. After evaporation the probe was etched in standard Al etchant (Merck 15435, Aluminum Etchant LE) for $10 \mathrm{~s}$, until the cantilever was transparent and thereby opening up the tip.

\section{NEAR-FIELD SCANNING OPTICAL MICROSCOPE}

In order to test the tips a near-field optical microscopical system was built in which these probes can be mounted. The apparatus is shown in Fig. 3.

The laser is a $8 \mathrm{~mW}$ HeNe laser focused onto a pinhole, which is subsequently imaged, using a Nikon extra long working distance (ELWD) objective with numerical aperature (NA) 0.5, on the base of the tip. The ELWD objective is necessary to provide space for the beam deflection system. The transmitted light through tip and sample is collected using a similar objective. Background light is further reduced using a pinhole in front of a Hamamatsu R1463 photomultiplier tube. The resulting setup thus consists of a confocal microscope with the probe inserted in its focus.
The sample is scanned using a three-axis piezo-electric scanner, consisting of a Photon Control $X-Y$ piezo flexure stage with a $200 \times 200 \mu \mathrm{m}^{2}$ range combined with a home built $10 \mu \mathrm{m}$ piezo stage for the $z$ direction. The scanner was designed to handle microscope object slides.

For detection of the bending of the cantilever a beam

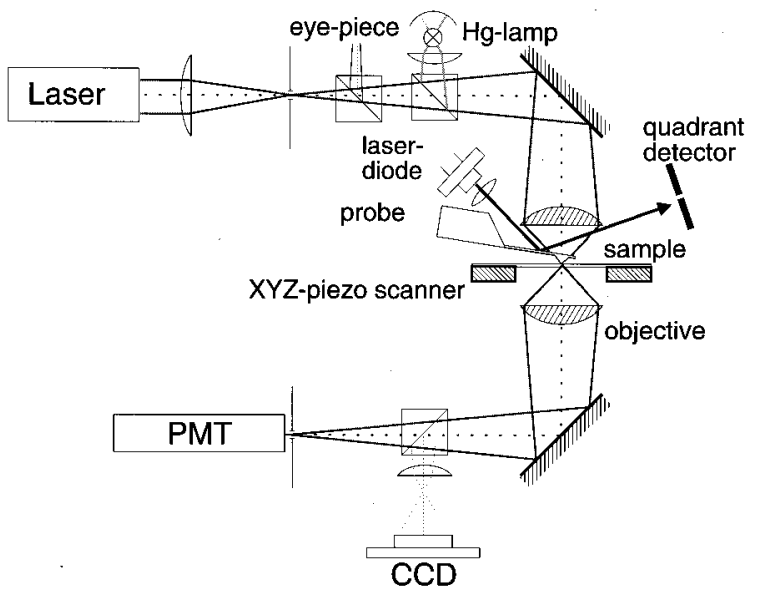

FIG. 3. Near-field optical microscope used with the microfabricated probes. 


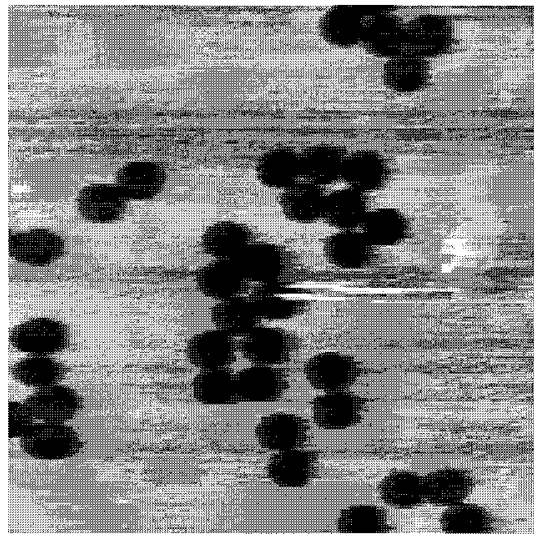

a

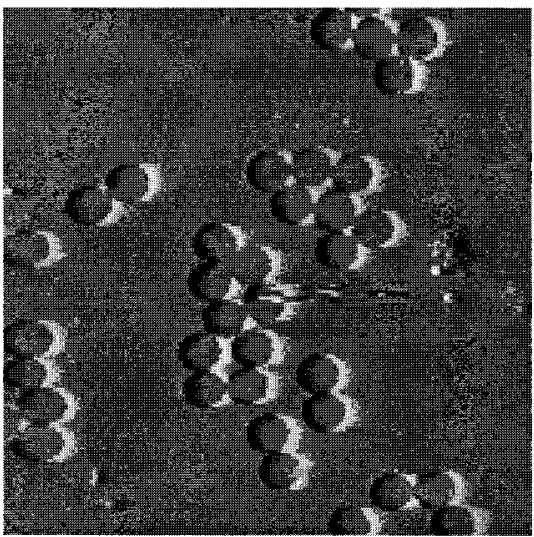

b

FIG. 4. Simultaneous images of a silver layer with $481 \mathrm{~nm}$ holes. Imaged with an uncoated tip (a) height ( $z$-piezo voltage); (b) deflection.

deflection technique is used. A laser diode at $780 \mathrm{~nm}$ is focused on the backside of the cantilever and its reflection is imaged onto a quadrant detector. The deflection signal, obtained by subtracting the signals from the vertically aligned quadrants, can be used as feedback signal for the $z$ piezo, as was done with the measurements shown here. The torsion signal, obtained by subtracting the signals from the horizontally aligned quadrants, displays the lateral force between tip and sample if the sample is scanned in the direction perpendicular to the cantilever.

In order to be able to align the optics and the sample with respect to the tip, a mercury lamp illuminates the sample which is imaged onto a charge coupled device (CCD) camera, displaying an area of about $100 \times 100 \mu \mathrm{m}^{2}$ of the sample and probe.

\section{EXPERIMENTAL RESULTS}

Figure 4 shows the results of a measurement of a $25 \mathrm{~nm}$ silver layer with $481 \mathrm{~nm}$ holes on a glass cover slip. The height image, displaying the $z$-piezo voltage, and the deflection image, displaying the feedback signal, clearly show the topography of the sample. From these results it can be concluded that the tip size is in agreement with Fig. 2(c) and that damage to the tip while scanning is not observed.
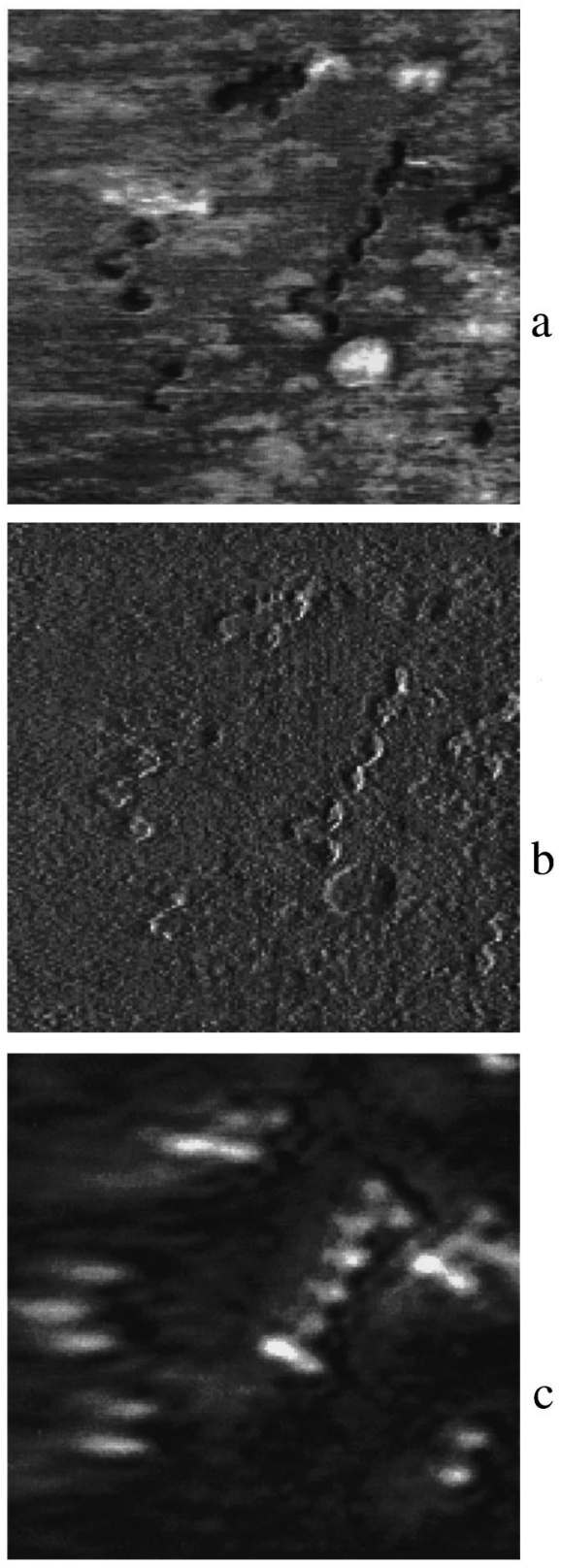

FIG. 5. Simultaneous images of a silver layer with $481 \mathrm{~nm}$ holes. Imaged with an Al-coated tip (a) height ( $z$-piezo voltage); (b) deflection; (c) optical transmission image.

The optical properties of the tip have been tested starting by checking the transmission properties and cone shape of the uncoated tips. By adjusting the light coupled into the tip the cone shape can be observed by looking at the light profile coupled out of the tip, e.g., when light coupled in at the left side of the tip it will be reflected towards the right side and exit the tip.

Figure 5 displays a measurement with a coated tip. Observed is that the height and deflection images are greatly influenced by the coating, resulting in tip convolution problems at the AFM images. The optical image in Fig. 5(c) shows that individual holes can be distinguished, with an optical resolution of about $300 \mathrm{~nm}$. 


\section{CONCLUSIONS}

A novel probe has been designed and developed. It has been demonstrated that the probes are well suited for AFM imaging and the optical properties agree with the design parameters. From the results it was shown that the aperture at the tip gives an optical resolution of $300 \mathrm{~nm}$ which is already below the diffraction limit, but can still be improved. We now focus on improvement of the $\mathrm{Al}$ coating and alternative ways to create a well defined aperture.

The apparatus was shown to be very powerful, giving simultaneous height and optical images. It opened up all known AFM contrast mechanisms for combination with near-field optical microscopy, resulting in a user friendly near-field microscope. The prospects of combining the microscope with tapping mode atomic force microscopy especially raises high expectations for future use of this type of probe.

Another advantage of this process is the batch fabrication of the probes, at present producing 448 probes per wafer, which stresses the promising role this type of probe will play in near-field optical microscopy.

\section{ACKNOWLEDGMENTS}

The authors would like to thank the members of the MESA research institute for their help, sharing their expertise and the useful discussions, especially Bert Otter for SEM imaging of the probes. This work was supported by the Dutch organization for fundamental research on matter (FOM).
${ }^{1}$ E. H. Synge, Philos. Mag. 6, 356 (1928).

${ }^{2}$ E. A. Ash and G. Nicholls, Nature 237, 510 (1972).

${ }^{3}$ E. Betzig, M. Isaacson, and A. Lewis, Appl. Phys. Lett. 51, 2088 (1987).

${ }^{4}$ D. W. Pohl, Adv. Opt. Electron Microsc. 12, 243 (1991).

${ }^{5}$ E. Betzig and J. K. Trautman, Science 257, 189 (1992).

${ }^{6}$ E. Betzig and R. J. Chichester, Science 262, 1422 (1993).

${ }^{7}$ M. H. P. Moers, H. E. Gaub, and N. F. van Hulst, Langmuir 10, 2774 (1994).

${ }^{8}$ H. A. Bethe, Phys. Rev. 66, 163 (1944).

${ }^{9}$ C. J. Bouwkamp, Philips Res. Rep. 5, 321 (1950).

${ }^{10}$ Aurora, Topometrix Corp.

${ }^{11}$ R. Toledo-Crow, Y. Chen, and M. Vaez-Iravani, Proc. SPIE 1639, 44 (1992).

${ }^{12}$ E. Betzig, P. L. Finn, and J. S. Weiner, Appl. Phys. Lett. 60, 2584 (1992).

${ }^{13}$ W. P. Ambrose, P. M. Goodwin, J. C. Martin, and R. A. Keller, Science 265, 364 (1994).

${ }^{14}$ P. J. Moyer and M. A. Paesler, NATO ARW Proc. NFO-1 Near Field Optics, 1992 (unpublished).

${ }^{15}$ A. Shchemelinin, M. Rudman, K. Lieberman, and A. Lewis, Rev. Sci. Instrum. 64, 3538 (1993).

${ }^{16}$ J. K. Trautman, J. J. Macklin, L. E. Brus, and E. Betzig, Nature 369, 40 (1994).

${ }^{17}$ M. Vaez-Iravani and R. Toledo-Crow, Appl. Phys. Lett. 63, 138 (1993).

${ }^{18}$ T. R. Albrecht, S. Akamine, T. E. Carver, and C. F. Quate, J. Vac. Sci. Technol. A 8, 3386 (1990).

${ }^{19}$ N. F. van Hulst, M. H. P. Moers, O. F. J. Noordman, R. G. Tack, F. B. Segerink, and B. Bölger, Appl. Phys. Lett. 62, 461 (1993).

${ }^{20}$ M. H. P. Moers, R. G. Tack, O. F. J. Noordman, F. B. Segerink, N. F. van Hulst, and B. Bölger, in Ref. 14, p. 79.

${ }^{21}$ F. Baida, D. Courjon, and G. Tribillon, in Ref. 14, p. 71.

${ }^{22}$ M. H. P. Moers, R. G. Tack, N. F. van Hulst, and B. Bölger, J. Appl. Phys. 75, 1254 (1994).

${ }^{23}$ N. F. van Hulst, M. H. P. Moers, and B. Bölger, J. Microsc. 171, 95 (1993).

${ }^{24}$ M. H. P. Moers, R. G. Tack, N. F. van Hulst, and B. Bölger, Scanning Microsc. 7, 789 (1993).

${ }^{25}$ M. Radmacher, P. E. Hillner, and P. K. Hansma, Rev. Sci. Instrum. 65, 2737 (1994). 